\title{
Preduodenal portal vein - a cause of antenatally diagnosed duodenal obstruction
}

\author{
Gattu H. ${ }^{1 *}$, Dhanani V. ${ }^{2}$, Susarla B. ${ }^{3}$, Sagar Ambati K. ${ }^{4}$ \\ DOI: https://doi.org/10.17511/ijpr.2020.i07.11 \\ 1* Harshitha Gattu, Neonatology Fellow, Department of Neonatology, Ankura Children's Hospital, Telangana, India. \\ 2 Vishakha Dhanani, Post-Graduate, Department of Neonatology, Ankura Children's Hospital, Telangana, India. \\ 3 Balaji Susarla, Senior Consultant, Department of Neonatology, Ankura Children's Hospital, Telangana, India. \\ ${ }^{4}$ Karuna Sagar Ambati, Senior Consultant, Department of Pediatric Surgery, Ankura Children's Hospital, Telangana, India.
}

Preduodenal portal vein (PDPV) is a rare congenital vascular anomaly in which the portal vein passes anterior to the duodenum rather than posteriorly. Generally asymptomatic, PDPV may rarely cause a duodenal obstruction in the newborn. It is usually associated with gastrointestinal tract, cardiac, pancreatic, as well as biliary tract anomalies or may, occur as a single isolated malformation. Till now, only a few cases have been reported with duodenal obstruction and associated anomalies. The present study report one such case of PDPV with multiple congenital anomalies. A full-term, oneday-old baby who had an antenatal history of polyhydramnios, presented to us with abdominal distension and non-bilious vomitings soon after birth. Surgical exploration revealed a hugely dilated stomach, multiple Ladd bands, malrotation of the small intestine, preduodenal portal vein, and an annular pancreas causing external duodenal compression. Intraoperative recognition of PDPV is important because iatrogenic injury during surgery can cause profuse hemorrhage from the vein and may cause damage to the biliary tract or duodenal wall. Prompt evaluation of associated cardiac anomalies is important before surgery. Duodenostomy anterior to the portal vein is the definitive treatment. Studies in animal models (AKR/J mice) have shown an autosomal recessive mode of inheritance.

Keywords: Preduodenal portal vein, Congenital vascular anomaly, Duodenostomy anterior

Corresponding Author

Harshitha Gattu, Neonatology Fellow, Department of Neonatology, Ankura Children's Hospital, Telangana, India.

Email: gattu.harshi@gmail.com
How to Cite this Article

To Browse

Gattu H, Dhanani V, Susarla B, Ambati KS

Preduodenal portal vein - a cause of antenatally diagnosed duodenal obstruction. Pediatric Rev Int J Pediatr Res. 2020;7(7):388-391.

Available From

https://pediatrics.medresearch.in/index.php/ijpr/arti cle/view/630

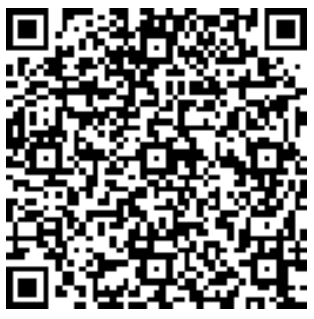

Manuscript Received 2020-10-02

Conflict of Interest No
Review Round 1 2020-10-12

Funding

Nil
Review Round 2 2020-10-20

Ethical Approval Yes
Review Round 3

Accepted 2020-10-29

Plagiarism $\mathbf{X}$-checker $9 \%$ ( 2020 by Harshitha Gattu, Vishakha Dhanani, Balaji Susarla, Karuna Sagar Ambati and Published by Siddharth Health
Research and Social Welfare Society. This is an Open Access article licensed under a Creative Commons Attribution 4.0 International License https://creativecommons.org/licenses/by/4.0/ unported [CC BY 4.0] 


\section{Introduction}

The preduodenal portal vein, which runs at the ventral side of the duodenum is a rare congenital anomaly in humans. The theory to account for the development of a preduodenal portal vein was put forth by His in 1885 [1], and an example of this anomaly in a pig embryo was reported by Begg in 1912 [2]. The first case report in humans was made by Knight in 1921 [3], who found this anomaly in a dissecting-room cadaver.

Since then, many cases have been reported in children and adults, as well as during surgery [4-6]. It is found sporadically during investigations of epigastric pain or other symptoms, such as vomiting or jaundice. This anomaly has a high association with other congenital anomalies, such as malrotation; situs inversus; pancreatic, duodenal, biliary system, and splenic anomalies; as well as dextrocardia. Its incidence is about 1 in 10000 $[7,8]$. PDPV is found in only $4 \%$ of cases with congenital obstruction [9]. Such an anomalous vein is a hazard in operations on the duodenum or biliary tract because of the dangers of inadvertent division or ligation.

\section{Case Report}

A one-day-old female outborn neonate weighing 2.5 $\mathrm{kg}$, born to a primigravida mother at 37 weeks of gestation by cesarean section, presented to us because of persistent non-bilious vomitings. An antenatal ultrasound scan was suggestive of duodenal atresia with polyhydramnios. On admission, the baby had respiratory distress. Per abdominal examination revealed upper abdomen distended with no visible peristalsis and no palpable lump. On cardiovascular examination, a systolic murmur of grade 3/6 intensity was heard, with the rest of the examination unremarkable. Clinical diagnosis of proximal duodenal obstruction with congenital heart disease was made. Routine supportive care and IV fluids were initiated as per unit protocol. The sepsis screen sent at admission was negative. X-ray erect abdomen done revealed a hugely distended stomach. 2DECHO done was suggestive of the infracardiac type of TAPVC. Delayed passage of meconium was present with the baby first passing stools at 45 hours of life. The pediatric surgeon's opinion was taken and as per his advice, barium meal with follow-through was done which showed the delayed passage of contrast in the small intestine.
Once hemodynamically stable, laparotomy was done which revealed a hugely dilated stomach, multiple Ladd bands, malrotation of the small intestine, a Preduodenal portal vein, and an annular pancreas causing external duodenal compression. Ladd bands were released, malrotation corrected and gastrojejunostomy was done. The intraoperative course remained uneventful.

Postoperatively, the baby was ventilated for a day following which she was extubated to nasal prongs oxygen. Enteral nutrition was started on the 3rd postoperative day, but unfortunately, the baby developed sepsis with blood culture growing Klebsiella. Hence, antibiotics were changed based on the sensitivity pattern. Baby improved clinically with the above management. Later, the Baby was referred to a higher center because of the infracardic type of TAPVC for surgical intervention.

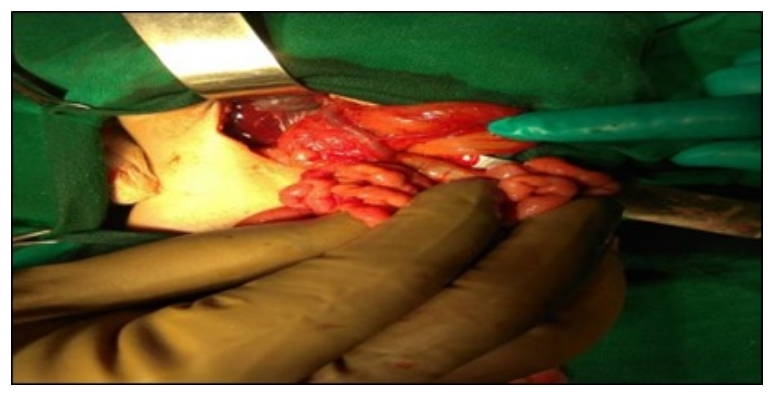

Fig-1: Preduodenal portal vein.

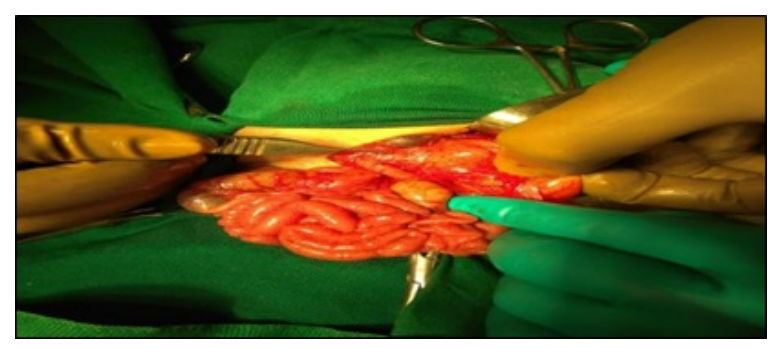

Fig-2: Malrotation of the small intestine and annular pancreas.

\section{Discussion}

In the 5th week of embryological development, the left and the right vitelline (omphalomesenteric) veins are distinguishable as separate entities. These veins form a plexus around the duodenum before entering the sinus venosus (Figure 3 ). This plexus comprises three cross-anastomoses between the left and the right vitelline veins - the superior anastomosis within the liver, the middle anastomosis posterior to the duodenum, and the inferior anastomosis that lies preduodenal. 
The 8 th to 12 th weeks of development see parts of this plexus atrophy (Figure 4). The superior and the inferior anastomosis atrophy, followed by the proximal part of the left and the distal parts of the right vitelline vein. This leaves the anastomotic network around the duodenum with one vessel, the portal vein. This vein lies posterior to the duodenum.

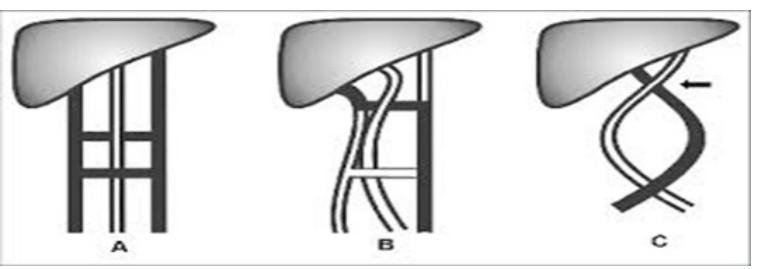

Fig-3: Schematic diagram of portal vein embryology. The left and right vitelline veins (colored black) form a ladder-like crossanastomosis around the primitive foregut seen in A. In B the middle posterior anastomosis remains patent (colored black) and the remainder of the anastomosis atrophies, resulting in the normal posterior position of the portal vein (black arrow).
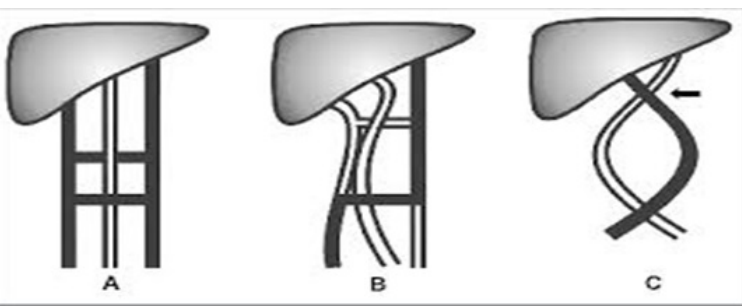

Fig-4: A schematic representation of PDPV development without malrotation. Note that the inferior preduodenal anastomosis remains patent $(B)$ resulting in a pre-duodenal position of the portal vein (black arrow) (C).
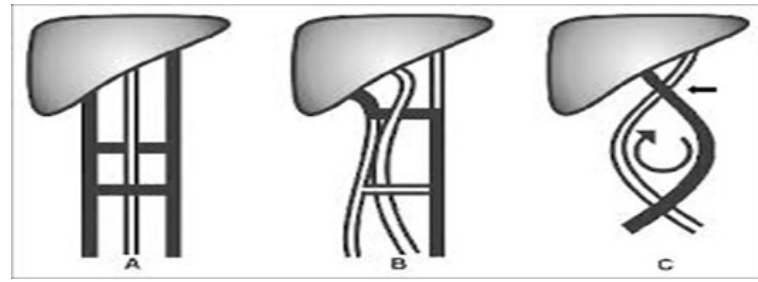

Fig-5: PDPV development with malrotation. Steps $A$ and $B$ are the same as in Fig. 2a; however, malrotation of the primitive gut results in a PDPV.

To explain the anomaly of a preduodenal portal vein, one of two possible events can occur (Figure 4 and 5).
Either the middle anastomosis atrophies and the inferior duodenal anastomosis remains patent resulting in a preduodenal position of the portal vein or the portal vein develops normally but associated malrotation of the gut leads to PDPV.

In our case, as there was associated malrotation of the intestine, the probable mechanism of development of PDPV could be as explained in figure 5.

PDPV as a cause for duodenal obstruction almost goes unrecognized on antenatal scans [6]. Postnatally, PDPV may be incidentally diagnosed or may lead to complete or partial duodenal obstruction, sometimes it may manifest later in adult life as gastric outlet obstruction or may be detected as an incidental finding during cholecystectomy $[8,9]$.

Clinical presentation in case of complete duodenal obstruction is vomiting since birth and feed intolerance. X-ray abdomen shows a double bubble sign suggestive of duodenal obstruction [3]. If the partial obstruction is present clinical features will be the failure to thrive, vomiting, and aspiration with time, such patients should undergo upper gastrointestinal contrast study [3].

\section{Conclusion}

It is not mandatory to diagnose PDPV preoperatively, eventually, all cases require laparotomy, but intraoperative recognition of PDPV is important because this may not be the sole cause of obstruction as seen in our case. It is important to look for the presence of associated anomalies like malrotation, presence of Ladd bands as well as the annular pancreas.

Palpation of the spleen intraoperatively is a must as the association of PDPV with polysplenia has been described. One must be watchful during surgery as complications such as iatrogenic haemorrhage can occur from the vein and damage to the biliary tract or distended duodenum. The patient should be taken for laparotomy after the condition of the infant is hemodynamically stable as the congenital duodenal obstruction is a relative surgical emergency.

Prompt evaluation for associated cardiac anomalies should be carried out. Duodenostomy anterior to the portal vein is the definitive treatment. Studies in animal models have shown an autosomal recessive mode of inheritance. 


\section{Reference}

01. His W. Human anatomy and embryology, Chapter III, Organ Histology, Vogel, Leipzig, Germany. in German. pp 1-260;1885. [Crossref]

02. Begg A. The anaomalous persistence in embryos of parts of the peri-intestinal rings formed by vitelline veins. Am J Anat. 1912;13(2)103-110. doi: 10.1002/aja.1000130203 [Crossref]

03. Knight HO. An anomalous portal vein with its surgical dangers. Ann Surg. 1921;74(6)697699.

doi: $10.1097 / 00000658-192112000-00004$ [Crossref]

04. Bower RJ, Trenberg JL. Preduodenal portal vein. J Pediatr Surg. 1972;7(5)579-584.

doi: $10.1016 / 0022-3468(72) 90216-3 \quad$ [Crossref]

05. Ishizaki YM, Tanaka T, Okuyama. Surgical implications of preduodenal portal vein in the adult. Arch Surg. 1994 Jul;129(7)773-5.

doi: $\quad$ 10.1001/archsurg.1994.01420310105020 [Crossref]

06. Ooshima L, Maruyama T, Ootsuki K, Ozaki M. Preduodenal portal vein in the adult. J Hepatobiliary Pancreat Surg. 1998;5(4)455-458. doi: $10.1007 / \mathrm{s} 005340050072$ [Crossref]
07. Tsuda Y, Nishimura K, Kawakami S, Kimura I, Nakano $Y$, Konishi J. Preduodenal portal vein and anomalous continuation of inferior vena cavaCT findings. J Comput Assist Tomogr. $1991 ; 15(4) 585-588$.

doi: $10.1097 / 00004728-199107000-00011$ [Crossref]

08. Grosfeld JL, Rescorla FJ. Duodenal atresia and stenosis reassessment of treatment and outcome based on antenatal diagnosis, pathologic variance, and long-term follow up. World J Surg. 1993;17(3)301-309.

doi: $10.1007 / B F 01658696$ [Crossref]

09. Yi SQ, Tanaka S,Tanaka A, Shimokawa T, Ru F, Nakatani T. An extremely rare inversion of preduodenal portal vein and common bile duct associated with multiple malformations, Report of an adult cadaver case with a brief review of the literature, Berlin. Anat Embryo. 2004;208(2)87-96.

doi: $\quad 10.1007 / \mathrm{s} 00429-003-0377-7 \quad$ [Crossref] 